\title{
Electrochemically Synthesized Bis(diimino)metal Coordination Nanosheets as Ultrastable Electrocatalysts for Hydrogen Evolution Reaction
}

\author{
Kuo-Hui Wu, ${ }^{\text {a, }}$ Jian Cao, ${ }^{\text {a }}$ Tigmansu Pal, ${ }^{\text {a.b }}$ Huaipu Yang, ${ }^{\text {a Hiroshi Nishihara*a,b }}$ \\ ${ }^{a}$ Department of Chemistry, Graduate School of Science, The University of Tokyo, 7-3-1, Hongo, Bunkyo- \\ ku, Tokyo 113-0033, Japan \\ ${ }^{\mathrm{b}}$ Research Center for Science and Technology, Tokyo University of Science, Chiba 278-8510, Japan \\ ${ }^{\dagger}$ Department of Chemistry, National Central University, 300 Jung-Da Rd. Jhong-Li 32001, Taiwan \\ E-mail: nisihara@rs.tus.ac.jp (H.N.)
}

Table of Contents

1. Materials and equipment

2. Experimental section of electrosynthesis

3. Electrochemistry of NiDI, CoDI and CuDI syntheses

4. Morphology characterizations

5. Powder X-ray diffraction and simulation of NiDI

6. Preparation of homemade RHE

7. Preparation of pretreated glassy carbon electrode 


\section{Materials and equipment}

Water used for synthesizing nanosheets was purified by a Millipore Milli-Q water system. HPLC-grade EtOH and acetone were purchased from KANTO CHEMICAL CO., INC. $\mathrm{Al}_{2} \mathrm{O}_{3}$ powder, $\mathrm{Ni}(\mathrm{OAc})_{2} \cdot 4 \mathrm{H}_{2} \mathrm{O}, \mathrm{CoCl} \mathrm{Cl}_{2}, \mathrm{Cu}(\mathrm{OAc})_{2}$ and $\mathrm{NaBF}_{4}$ were also purchased from commercial sources and used without further purification.

The electrosyntheses were conducted by HOKUTO DENKO HZ-3000 automatic polarization system. Pt coil, ITO and carbon paper were purchased from commercial sources. Pt coil, ITO and carbon papers were thrice rinsed by water and ethanol and fully dried by vacuum before using. A homemade $\mathrm{Ag} / \mathrm{AgCl}$ electrode was used as a reference electrode. Electrochemical measurements were carried out using ALS 650DT electrochemical analyzer (BAS. Co. Ltd.) or HOKUTO DENKO HZ-3000 automatic polarization system.

Optical microscope images were taken using VHX-100 (Keyence Corporation). Scanning electron microscopy (SEM) images were taken by JEOL JSM-7400FNT. X-ray photoelectron spectroscopy (XPS) measurements were conducted by using ULVAC-PHI PHI 5000 VersaProbe spectrometer. Al K $\alpha(20 \mathrm{kV}, 100 \mathrm{~V})$ was used as the X-ray source, and the beam was focused on a $100 \mu \mathrm{m}^{2}$ area. The XPS data were analyzed by using MultiPak Software and standardized binding energy by using C 1 s peak at $284.8 \mathrm{eV}$. Powder X-ray diffraction (PXRD) was recorded using synchrotron radiation at Beamline BL44B2 at Super Photo ring-8 GeV (SPring-8) with a wavelength of 0.108 nm.

\section{Experimental section of electrosynthesis}

All the synthetic procedures of nanosheets were carried out under an argon atmosphere.

Electrosynthesis of NiDI: The precursor solution $(10 \mathrm{~mL})$ which contained $\mathrm{HAB} \cdot 3 \mathrm{HCl}(2.2 \mathrm{mg}, 7.9 \mu \mathrm{mol})$, $\mathrm{Ni}(\mathrm{OAc})_{2} \cdot 4 \mathrm{H}_{2} \mathrm{O}(2.2 \mathrm{mg}, 8.8 \mu \mathrm{mol}), \mathrm{NaBF}_{4}(0.109 \mathrm{~g}, 1 \mathrm{mmol}), 0.63 \mathrm{M}$ ammonia solution $(0.3 \mathrm{~g})$ was prepared in glove box. Then electrosyntheses were conducted in the precursor solution by a three-electrode cell with ITO, carbon paper or glassy carbon electrode as working electrode, a Pt coil as counter electrode and an $\mathrm{Ag} / \mathrm{AgCl}$ reference electrode. By applying a constant potential $(0.58 \mathrm{~V}$ vs. $\mathrm{Ag} / \mathrm{AgCl})$ for $3 \mathrm{~min}$, nanosheets gradually form on the working electrode under successive electrolysis.

Electrosynthesis of CoDI: The precursor solution $(10 \mathrm{~mL})$ was made of $\mathrm{HAB} \cdot 3 \mathrm{HCl}(2.2 \mathrm{mg}, 7.9 \mu \mathrm{mol}), \mathrm{CoCl}_{2}$ (6 mg, $46 \mu \mathrm{mol}), \mathrm{NaBF}_{4}(0.109 \mathrm{~g}, 1 \mathrm{mmol}), 0.63 \mathrm{M}$ ammonia solution $(1.5 \mathrm{~g}, 0.965 \mathrm{mmol})$ under an argon atmosphere. The same electrochemical cell setting was used, and CoDI coated substrate can be acquired after applying a constant potential at $0.21 \mathrm{~V}$ vs. $\mathrm{Ag} / \mathrm{AgCl}$ within $3 \mathrm{~min}$.

Electrosynthesis of CuDI: The preparation of CuDI is similar to NiDI and CoDI. HAB $\cdot 3 \mathrm{HCl}(2.2 \mathrm{mg}, 7.9 \mu \mathrm{mol})$, $\mathrm{Cu}(\mathrm{OAc})_{2}(6.0 \mathrm{mg}, 33 \mu \mathrm{mol})$ and $\mathrm{NaBF}_{4}(0.109 \mathrm{~g}, 1 \mathrm{mmol})$ were dissolved in $0.63 \mathrm{M}$ ammonia $(1.5 \mathrm{~g}, 0.965 \mathrm{mmol})$ aqueous solution $(10 \mathrm{~mL})$. A constant potential of $0.185 \mathrm{~V}$ vs. $\mathrm{Ag} / \mathrm{AgCl}$ was applied for $3 \mathrm{~min}$ and CuDI can form during the electropolymerization.

CONASH modified conductive substrates were brought out from the argon atmosphere and immersed into deionized water and ethanol separately three times to remove metal ions and organic residues. The remaining solvents on the electrode surface were blown away by argon or $\mathrm{N}_{2}$ flow. Then as-prepared modified electrodes were dried under vacuum for 6 hours before characterizations. The sample of NiDI for powder XRD was prepared in another way. The precursor solution was prepared as mentioned above. A long piece of carbon paper was used as a working electrode, and electrosynthesis was conducted under stirring by applying the same reaction potential for 3 hours. Black powder can form and be detached from carbon paper because of stirring, then was collected after electropolymerization. The powder was rinsed by water, ethanol, and acetone. At last, it was dried under vacuum for 6 hours. 


\section{Electrochemistry of NiDI, CoDI and CuDI syntheses}

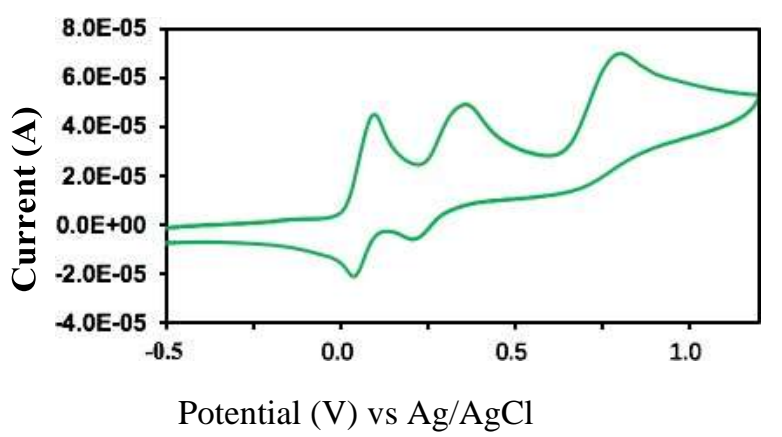

Figure S1. The cyclic voltammogram of $\mathrm{HAB} \cdot 3 \mathrm{HCl}$

Figure $\mathrm{S} 1$ shows the cyclic voltammogram of $\mathrm{HAB} \cdot 3 \mathrm{HCl}$, which is dissolved in the water. Three oxidation peaks are observed at $0.10 \mathrm{~V}, 0.36 \mathrm{~V}$ and $0.80 \mathrm{~V}$ vs. $\mathrm{Ag} / \mathrm{AgCl}$ and three corresponding reduction peaks were at $0.04 \mathrm{~V}$, $0.22 \mathrm{~V}$ and $0.67 \mathrm{~V}$. These redox peaks can be attributed to the redox activity of HAB.

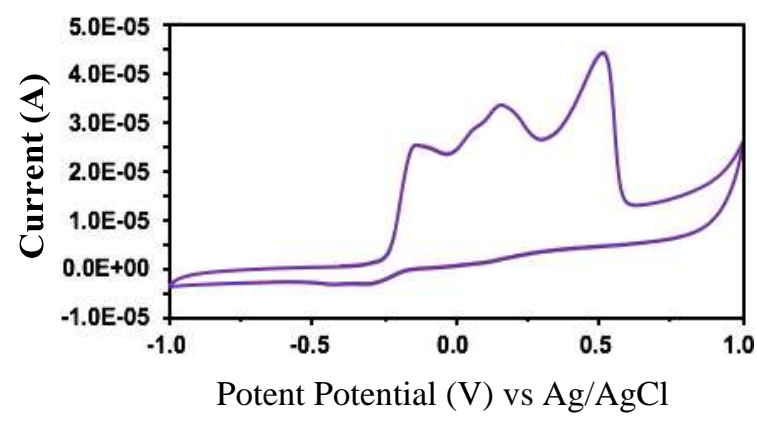

Figure S2. The cyclic voltammogram of precursor solution for preparing NiDI (the first cycle).

Figure S2 shows the cyclic voltammogram of precursor solution for preparing NiDI. Multi-oxidation signals can be observed but the shape was different from the pure ligand electrolyte solution. The coordination reaction had already started, and the partially coordinated ligand showed a different redox response with Figure S1. Besides, the number of reduction peaks decreased, and the shape and intensities also changed a lot. This phenomenon can be attributed to the ligands near the working electrode surface were consumed after oxidizing and coordinating to form low redox-active species and new ligands cannot be supplied timely.

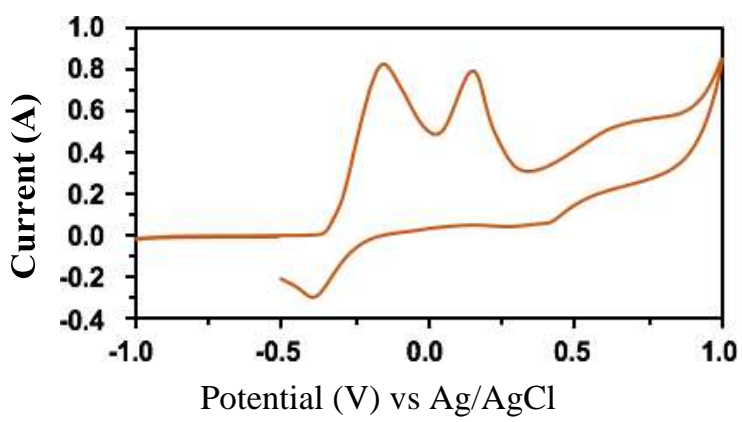

Figure S3. The cyclic voltammogram of precursor solution for preparing CoDI (the first cycle).

Figure S3 shows the cyclic voltammogram of precursor solution for preparing CoDI. Two oxidation peaks at -0.2 $\mathrm{V}$ and $0.2 \mathrm{~V}$ became much more distinct and one reduction disappeared compared with Figure S1. Different metal ions can show different situations in coordinating with ligands so that the cyclic voltammograms for each condition also became unique. Since the reactants near the working electrode were consuming gradually during the oxidation, the decrescent intensities of reduction peaks are reasonable. 


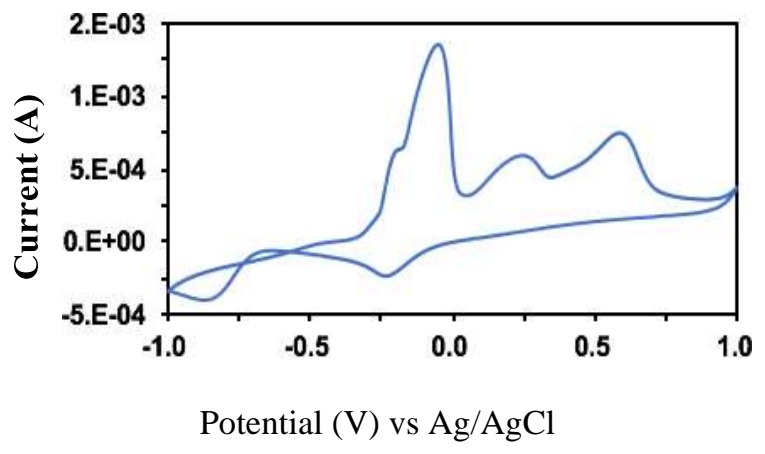

Figure S4. The cyclic voltammogram of precursor solution for preparing CuDI (the first cycle).

Figure S4 shows the cyclic voltammogram of precursor solution for preparing CuDI. The CV curve also showed distinctive characters with the previous two results. And the oxidation of ligand and coordination between ligand and metal can also be judged by reduction peaks. 


\section{Morphology characterizations}

The sheet-like morphology of CONASH synthesized by electropolymerization was also determined by electron microscopy. Figure S5 shows the scanning electron microscopic (SEM) image of NiDI on the ITO substrate. From Figure S5(a), flat and clean sheet-like morphology and large two-dimensional network can be observed in CONASH modified domain. Exfoliated nanosheets also existed on the bare ITO surface. At the same time, some regions of the CONASH modified area showed peel-off morphology Figure S5(b) and implied the formation of exfoliated nanosheets. The zoom-in images were taken and folded nanosheets on the edge revealed that the electrosyntheized nanosheet exhibited rigid and free-standing nature. Electrosynthesized CoDI and CuDI samples on ITO substrate also exhibited similar surface conditions with NiDI.

a

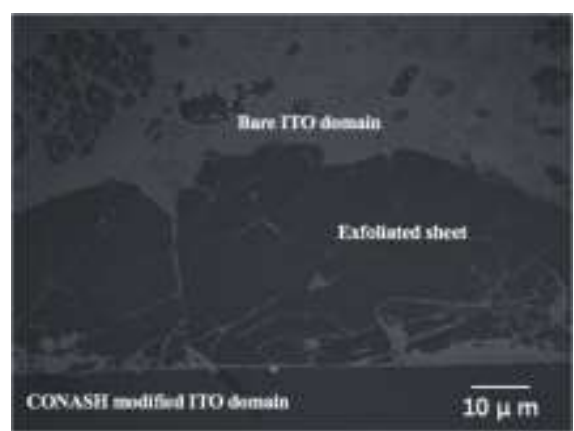

b

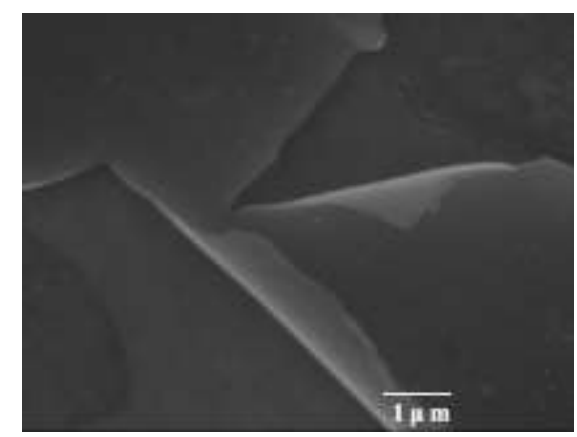

Figure S5. Scanning electron microscopic images of NiDI CONASH-modified ITO. 


\section{Powder X-ray diffraction and simulation of NiDI}

The amount of electrosynthesized CONASHs on the conductive substrate surface was too little to collect for powder XRD measurements. So that increasing the surface of the working electrode and using stirring to accelerate the reactant dispersion near the electrode surface are applied during the preparation of the powder sample. And the solution flow through the electrode surface also made synthesized CONASHs easily detach from the substrate surface and resulted in a large number of powder samples. Powder XRD result of NiDI was gotten from SPring-8 and shown as a purple line in Figure S6. Low crystallinity made the peaks become broaden. This phenomenon is reasonable because of the accelerated formation process of CONASHs by electrosynthesis. A high reaction rate may cause a disorder in structure arrangement or the formation of defects. Therefore, low crystallinity becomes a common case in electrosyntheses of CONASHs. Some conducting polymers fabricated by electrosyntheses also showed low crystallinities.

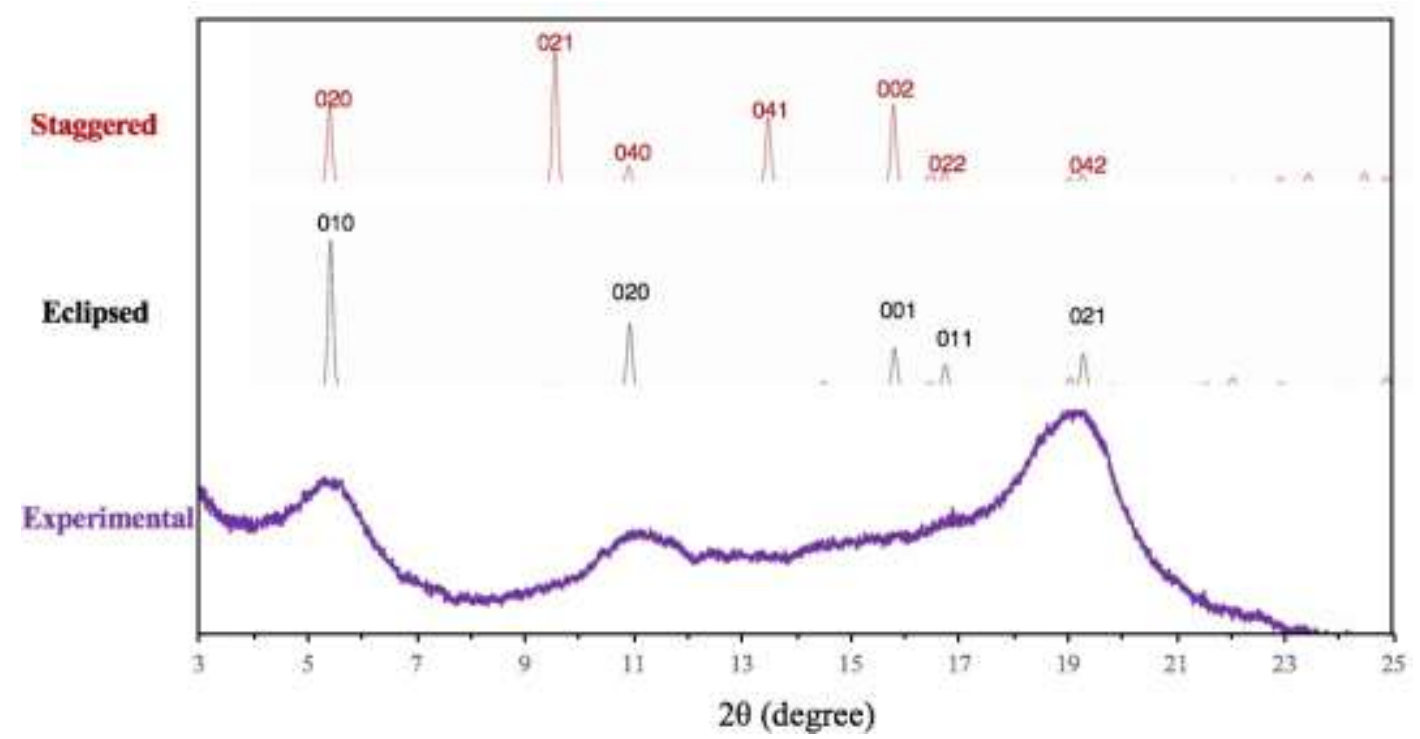

Figure S6. Powder X-ray diffraction comparison between experimental data with simulation results from staggered and eclipsed mode of NiDI.

(a) Eclipsed

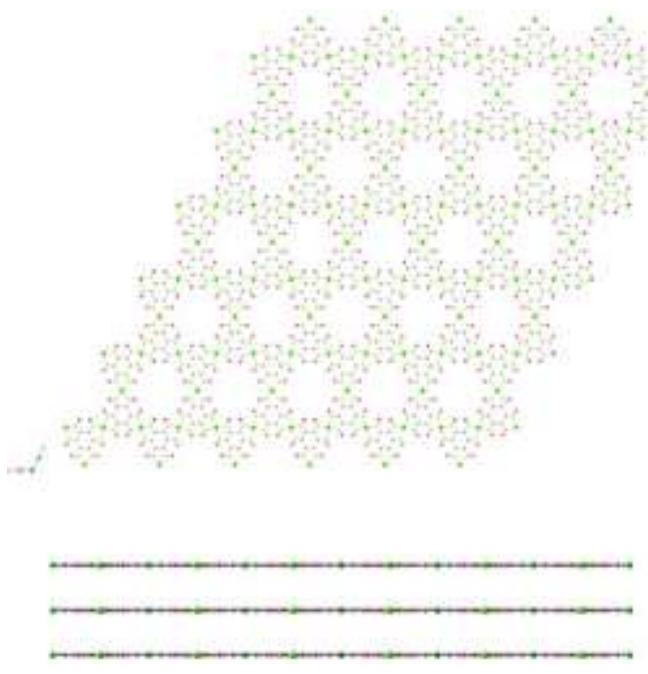

(b) Staggered

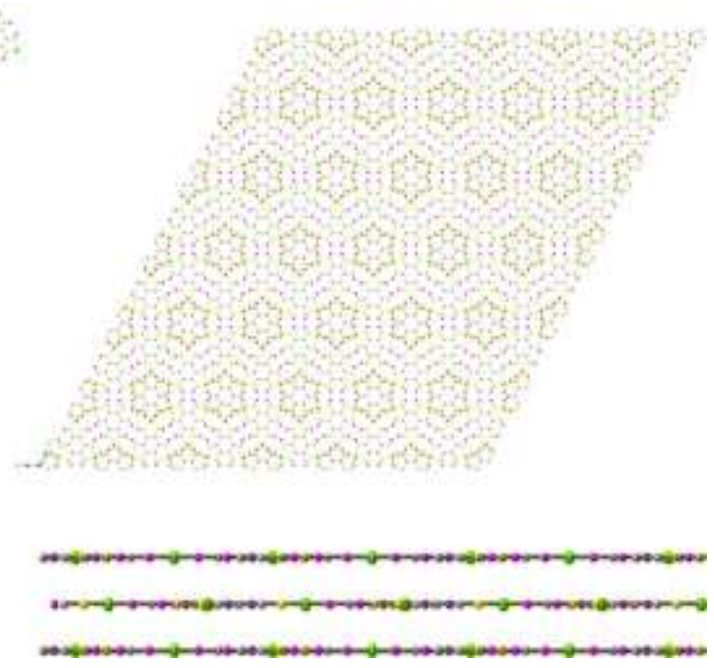

Figure S7. Top and side views of eclipsed structure (a) and staggered (b) structure of NiDI. 


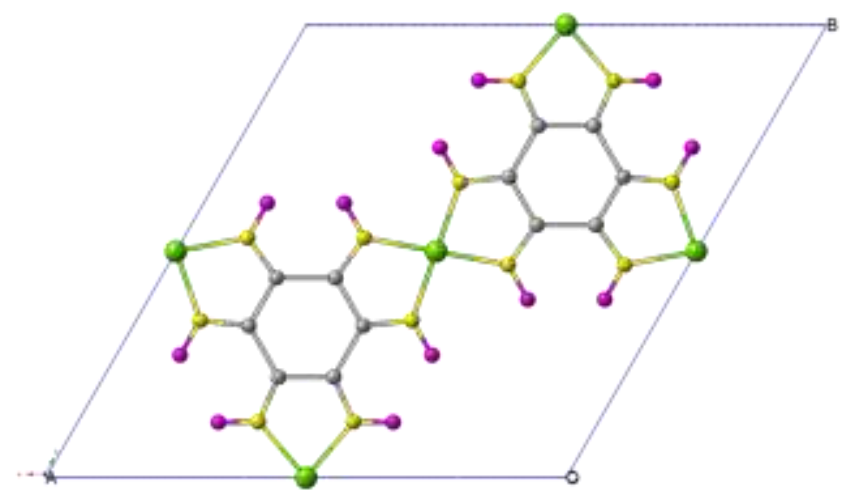

Figure S8. Unit cell structure of NiDI in eclipsed structure, C, H, N and Ni atoms are denoted as gray, pink, yellow and green dots.

The diffraction patterns of staggered and eclipsed packing modes were simulated and were also indicated in Figure S7. They have $P 6_{3} / \mathrm{mmm}$ and $P 6 / \mathrm{mmm}$ space groups, respectively, and top views and side views were both showed in Figure 3-4. According to the comparison among simulated results and experimental results, NiDI revealed coherence with eclipsed structure as showed in Figure S7 (a). Main peaks with $2 \theta$ equal to $5.46^{\circ}, 10.98^{\circ}$, and $19.22^{\circ}$ can be assigned from (010), (020) and (021) respectively. And detailed parameters of the unit cell were also determined as $\mathrm{a}=\mathrm{b}=13.31 \AA$ and $\mathrm{c}=0.34 \mathrm{~nm}$ (Figure S8).

\section{Preparation of homemade RHE}

During the electrocatalytic test under acidic condition, $\mathrm{Ag} / \mathrm{AgCl}$ often showed inconsistent potential because of the influence from the acidic solution penetration to porous glass. Besides, the $\mathrm{pH}$ value obtained from the $\mathrm{pH}$ meter under the high acidity showed non-negligible error. Therefore, for getting a reliable valuation of catalytic perfor-

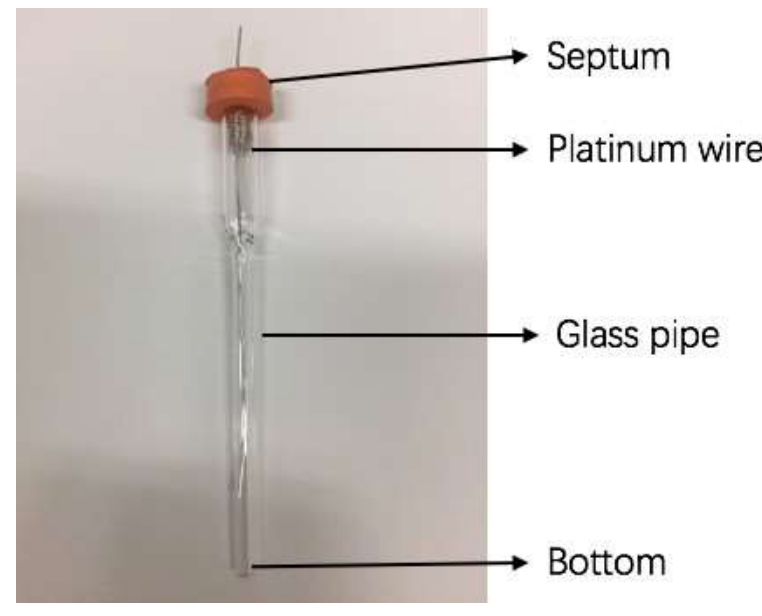

Figure S9. The photo of homemade reversible hydrogen electrode. mance in acidic solution, $\mathrm{Ag} / \mathrm{AgCl}$ was not suitable and a homemade reversible hydrogen electrode (RHE) was prepared.

The photo of this homemade RHE was shown in Figure S9. The preparation of RHE before the electrocatalytic test was introduced as follows. At first, an acidic solution for electrocatalytic measurement was prepared by using $\mathrm{H}_{2} \mathrm{SO}_{4}$ as an electrolyte. Part of this solution was injected and filled with the glass pipe from the bottom. Then electrolysis in the same acidic solution was conducted by using RHE as cathode and platinum coil as an anode. By applying constant potential as $-3.5 \mathrm{~V}$ for 60 seconds, the solution in the RHE pipe was hydrogen saturated and the hydrogen bubble can aggregate at the top of the RHE. After that, the prepared electrode can act as standard RHE for this acidic solution and show reliable stability during the experiments. 


\section{Preparation of pretreated glassy carbon electrode}

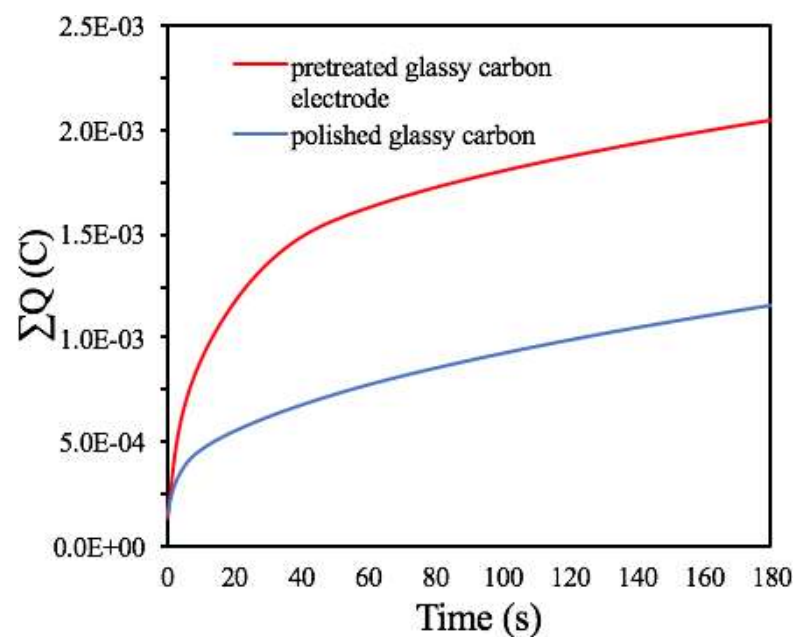

Figure S10. The coulomb chronometry (controlled potential coulometry) results of NiDI electrosyntheses on pretreated glassy carbon and polished.
Glassy carbon electrode surface reveals hydrophobic nature and therefore has a bad affinity with hydrophilic CONASHs. One way to activate the glassy carbon surface was anodic polarization. In this research, polished glassy carbon was pretreated by scanning cyclic voltammetry in $0.1 \mathrm{M} \mathrm{H}_{2} \mathrm{SO}_{4}$ from $0 \mathrm{~V}$ to $2.2 \mathrm{~V}$ (vs. $\mathrm{Ag} / \mathrm{AgCl}$ ) for 25 cycles with a scan rate of $0.1 \mathrm{~V} / \mathrm{s}$. And similar double-layer capacitances can be gotten after polish and pretreatment to different glassy carbon electrodes. Compared with polished glassy carbon, more electrochemical reactions can proceed on pretreated glassy carbon electrode surfaces with the same electrosynthesis conditions (Figure S9). Therefore, pretreated glassy carbon electrodes can possess and hold more catalysts. Besides, according to XPS, the chemical structures of electrosynthesized CONASHs on pretreated glassy carbon electrodes kept the same as the results gotten from other electrodes.

It means that pretreated glassy carbon can be a good supporting electrode for electrocatalytic research especially for NiDI, CoDI and CuDi which can be electrosynthesized and modified on electrodes directly, and the measurements were also reliable. This research proved that the NiDI indeed showed superior catalytic efficiency with an overpotential of $227 \mathrm{mV}$ (vs. RHE) at the current density of 10 $\mathrm{mA} / \mathrm{cm}^{2}$ in $0.5 \mathrm{M} \mathrm{H}_{2} \mathrm{SO}_{4}$. This value was also the best electrocatalytic value in hydrogen evolution reaction compared with reported coordination nanomaterials until now. Low onset potential and extremely high exchange current density of NiDI also indicated that this material possesses high intrinsic catalytic activity.

Although the structures of single molecular complexes of NiDI, CoDI and CuDI were reported many years ago, recently NiDI's single molecular complex $\mathrm{Ni}_{2}\left[\mathrm{C}_{6} \mathrm{H}_{4}(\mathrm{NH})_{2}\right]_{2}$ was studied and showed good performance in catalytic application in hydrogen evolution reaction ${ }^{2}$. Therefore, NiDI, which can arrange a large amount of Ni-diimino complex motifs into one plane and correlated them in a high $\pi$-conjugation system with redox-active ligands, had been expected to become a potential candidate as electrocatalyst for hydrogen evolution reaction because of its universal catalytic centers and high conductivity. Besides, good affinities between CONASHs and electrodes and perfect covers on substrate surfaces can be achieved by electrosynthesis. These features in the new method can efficiently reduce the system electroresistance and also improve the catalytic properties. Therefore, intrinsic structures and appropriate method in electrocatalyst preparation can be regarded as key factors, leading to NiDI's high catalyst efficiency in hydrogen evolution reaction

Furthermore, Tafel slopes of NiDI, and CoDI and CuDI were similar which meant that their catalytic reaction mechanisms are also similar. Taken the high concentration of proton in solution into consideration, the catalytic reaction mechanism with NiDI, CoDI and CuDI as catalysts can be attributed as following Volmer-Heyrovsky HER mechanism. The detailed procedure was described by chemical equations (1) and (2).

$$
\begin{gathered}
\mathrm{H}_{3} \mathrm{O}^{+}+\mathrm{e}^{-} \rightarrow \mathrm{H}_{\text {ads }}+\mathrm{H}_{2} \mathrm{O} \\
\mathrm{H}_{\text {ads }}+\mathrm{H}_{3} \mathrm{O}^{+}+\mathrm{e}^{-} \rightarrow \mathrm{H}_{2}+\mathrm{H}_{2} \mathrm{O}
\end{gathered}
$$

